\title{
An alpha-fetoprotein-negative hepatoid adenocarcinoma of the gallbladder with squamous differentiation
}

\author{
Xiaohui Qian ${ }^{1,2,3,4,5}$, Dongkai Zhou ${ }^{1,2,3,4,5}$, Bingqiang Gao ${ }^{1,2,3,4,5}$, Weilin Wang ${ }^{1,2,3,4,5}$ \\ ${ }^{1}$ Department of Hepatobiliary and Pancreatic Surgery, The Second Affiliated Hospital, Zhejiang University School of Medicine, Hangzhou 310009 , \\ China; ${ }^{2}$ Key Laboratory of Precision Diagnosis and Treatment for Hepatobiliary and Pancreatic Tumor of Zhejiang Province, Hangzhou 310009 , \\ China; ${ }^{3}$ Research Center of Diagnosis and Treatment Technology for Hepatocellular Carcinoma of Zhejiang Province, Hangzhou 310009 , China; \\ ${ }^{4}$ Clinical Medicine Innovation Center of Precision Diagnosis and Treatment for Hepatobiliary and Pancreatic Disease of Zhejiang University, \\ Hangzhou 310009, China; ${ }^{5}$ Clinical Research Center of Hepatobiliary and Pancreatic Diseases of Zhejiang Province, Hangzhou 310009 , China \\ Correspondence to: Weilin Wang. Department of Hepatobiliary and Pancreatic Surgery, The Second Affiliated Hospital, Zhejiang University School of \\ Medicine, No. 88 Jiefang Road, Hangzhou 310009, China. Email: wam@zju.edu.cn.
}

Submitted Oct 30, 2019. Accepted for publication Nov 12, 2019.

doi: 10.21037/hbsn.2019.12.03

View this article at: http://dx.doi.org/10.21037/hbsn.2019.12.03

During a routine health examination, a 61-year-old male was incidentally found to have a neoplasm spreading beyond the gallbladder and into the liver on abdominal ultrasound. The ultrasound demonstrated a mixedecho mass, measuring $7.6 \mathrm{~cm} \times 4.8 \mathrm{~cm}$ in diameter, within the capsuled wall of the gallbladder and adhering to the liver. The patient was subsequently admitted to our hospital. His medical, family, and personal history were unremarkable, and serology tests for hepatitis B and $\mathrm{C}$ were both negative. Laboratory tumor marker investigations revealed increases in carcinoembryonic antigen (104.7 ng/mL; normal: $0-5 \mathrm{ng} / \mathrm{mL})$, carbohydrate antigen (CA) $125(421.3 \mathrm{U} / \mathrm{mL}$; normal: 0-37 U/mL), CA 242 (220.4 U/mL; normal: 0-20 U/mL), and CA19-9 (2,118.4 U/mL; normal: 0-27 U/mL) levels. Plasma alpha-fetoprotein (AFP) was within the normal range. An enhanced computed tomography (CT) scan revealed a mass in the gallbladder bed with enlarged hilar lymph nodes (Figure 1A,B,C). A cholecystectomy was subsequently performed, along with a liver and lymph node dissection. Histological findings revealed poorly differentiated cells with abundant eosinophilic cytoplasm (Figure 1D,E), and positive staining for cytokeratin-7 (CK7) and hepatocyte specific protein (HepPar-1) by immunohistochemistry (Figure $1 F G$ ), suggesting that these cells were hepatoid adenocarcinoma (HAC). The tumor location, radiological features, and positivity for HepPar-1 suggested a diagnosis of HAC of the gall bladder (TNM stage: T3N1M0). In addition, the tumor revealed focal squamous epithelial differentiation and was positive for $\mathrm{P} 40$ and P63 proteins (Figure 1H,I). The postoperative course was uneventful, and the patient was discharged 10 days later. During the 5-month follow-up, the patient received intravenous paclitaxel as adjuvant chemotherapy, without recurrence, and was able to resume normal activities.

HAC, presenting the morphological and immunohistochemical features of hepatocellular carcinoma (HCC), is a rare type of extrahepatic malignant epithelial neoplasm. The majority of HACs occur in the stomach, which may be due to the fact that the stomach and liver originate from the same blastoderm (1). In addition, HAC is often AFP positive, whereas in this case, it was negative (2). Primary HAC of the gallbladder is exceptionally rare. To the best of our knowledge, we have reported the first case of squamous differentiated AFP-negative HAC of the gallbladder. Patients suffering from HAC of the gallbladder have a poor prognosis; therefore, correctly identifying the hepatoid component within gallbladder cancer and providing suitable treatment for patients is crucial. The main differential diagnosis of HAC of the gallbladder is HCC, as it is difficult to determine whether HAC is invading into the liver or the gallbladder when HAC invades deeply into the liver. Gathering medical history and the clinical presentation data of patients is essential for aiding the diagnosis, as HCC often arises in a cirrhotic liver associated with risk factors including hepatitis virus 

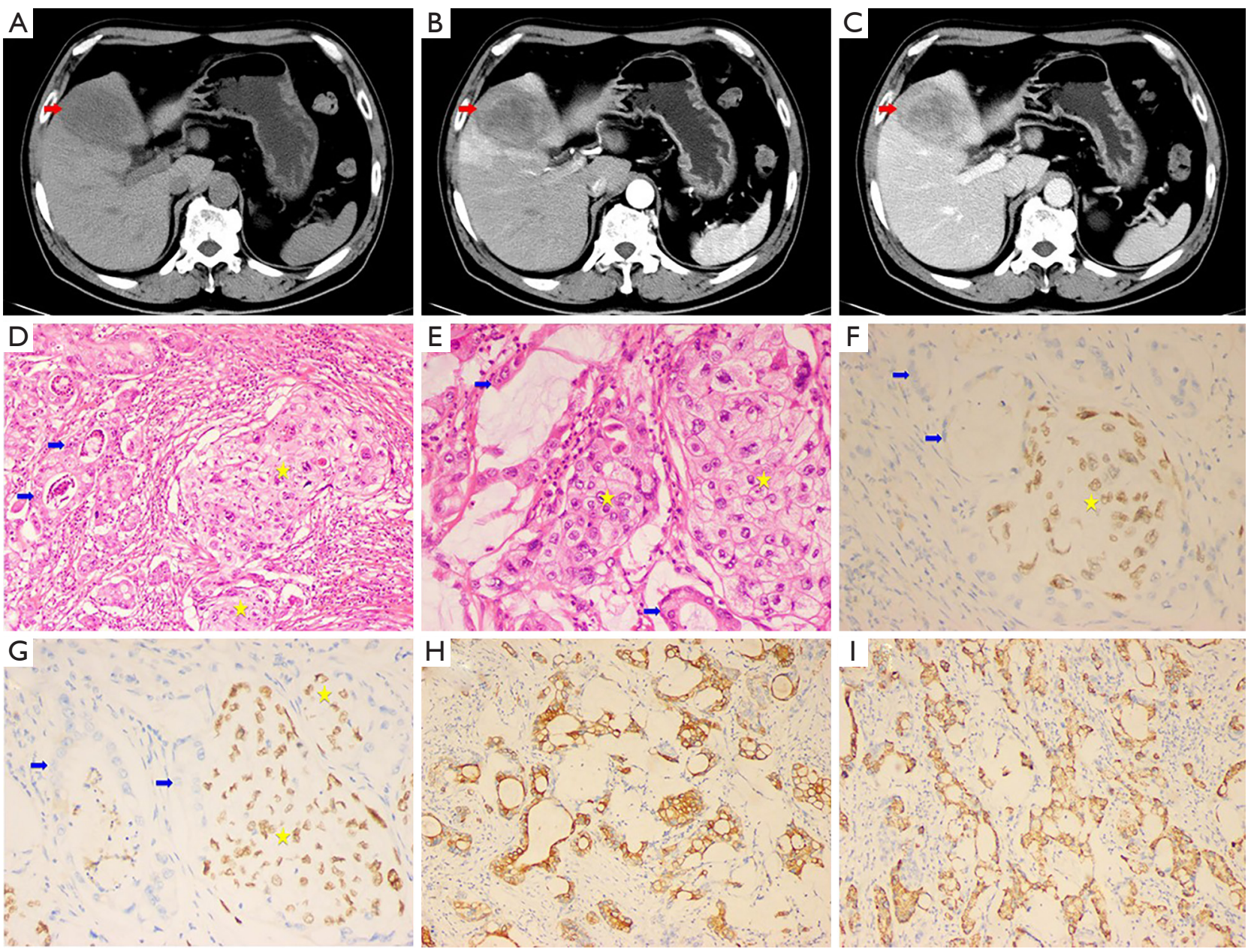

Figure 1 Computed tomography (CT), histological, and immunohistochemical images. Contrast-enhanced CT images (A,B,C) showed a mass on the gallbladder measuring $32 \mathrm{~mm} \times 23 \mathrm{~mm}$, with heterogeneous enhancement of the gallbladder (red arrows). Histological analysis revealed that most of the tumor presented with glandular structures, as indicated by blue arrows, while a nested growth pattern could be found focally, as shown by asterisks (D,E; hematoxylin-eosin staining, 200× magnification). Immunohistochemistry analysis showed P63 and $\mathrm{P} 40$ positive staining for these nested areas, and negative staining for the glandular structures, indicating squamous differentiation (F, G; 200× magnification). Cytokeratin-7 and hepatocyte specific protein were positive (H,I; 200× magnification).

infection and alcohol abuse (3). The therapeutic approaches for HAC and HCC differ, especially in patients with a noncirrhotic liver. As with the case described here, ultrasound and CT revealed that the tumor spanned beyond the gallbladder wall to the adjacent liver. The patient tested negative for AFP and had no history of alcohol abuse, hepatitis, or hepatic cirrhosis, and thus, prior to surgery, we considered it possible that the origin of this tumor was the gallbladder.

Obtaining an accurate diagnosis of HAC of the gallbladder prior to surgery is extremely difficult (4). The final diagnosis is determined by a histopathological examination of specimens obtained from patients by surgery or biopsy. HAC is characterized by hepatoid differentiated cells, arranged in a trabecular pattern, with abundant eosinophilic cytoplasm (5), as demonstrated by this case. Additional immunohistochemistry is essential for the differential diagnosis. Diffuse CK7 or CK19 positivity suggests the presence of adenocarcinoma. Although the latest studies have challenged the specificity and sensitivity of HepPar-1 and glypican-3, they have served as markers of hepatic differentiation in cytological specimens (6). AFP positivity in the tumor and/or increased levels in the serum are associated with these tumor cells, particularly in 
stomach tumors, demonstrating the importance of AFP in accurate HAC diagnosis (7). In this case, the combination of the location of the tumor, hepatic differentiation noted in the adenocarcinoma cytological specimen, and CD7 and HepPar-1 positivity supported a diagnosis of HAC of the gallbladder. Interestingly, the tumor was positive for $\mathrm{P} 40$ and P63, which means that HAC has the ability to show squamous differentiation. Squamous cell carcinomas easily become large masses due to their ability to proliferate. In addition, adenosquamous carcinomas of the gallbladder are less able to metastasize in comparison with adenocarcinoma tumors, and they tend to invade adjacent organs rather than metastasizing to distal organs (8). In the present case, the tumor was large and had metastasized to the liver and lymph nodes only, which may be associated with squamous differentiation. As a result, the tumor was finally classified as T3N1M0. The clinical significance of HAC with squamous differentiation remains unknown, and further study is required to understand this.

Most cases of HAC of the gallbladder undergo surgical treatment, involving radical resection of the mass, and we recommend a resection that includes the gallbladder, segments IV A and V of the liver (wedge of the gallbladder bed), and lymph nodes to achieve R0 resection of the tumor if feasible. Adjuvant or palliative chemotherapy (paclitaxel alone or in combination with 5 -fluorouracil) is suggested for inoperable or metastatic HACs of the stomach (9). Other treatments, such as radiotherapy and immunotherapy, have not been performed for HAC of the gallbladder.

In summary, we illustrate a rare case of AFP-negative HAC of the gallbladder with squamous differentiation. It was difficult to make an accurate preoperative diagnosis of HAC of the gallbladder due to limited radiological characteristics and a lack of tumor markers. We highlight the importance of histological and immunohistochemical investigations of HAC. Although HAC of the gallbladder is extremely rare, it is important to be aware of its existence to avoid misdiagnosing HAC as advanced HCC. More studies are needed to demonstrate the significance of HAC with squamous differentiating ability.

\section{Acknowledgments}

None.

\section{Footnote}

Conflicts of Interest: The authors have no conflicts of interest to declare.

Ethical Statement: The authors are accountable for all aspects of the work in ensuring that questions related to the accuracy or integrity of any part of the work are appropriately investigated and resolved. Written informed consent was obtained from the patient for publication of this manuscript and any accompanying images.

\section{References}

1. Terracciano LM, Glatz K, Mhawech P, et al. Hepatoid adenocarcinoma with liver metastasis mimicking hepatocellular carcinoma: an immunohistochemical and molecular study of eight cases. Am J Surg Pathol 2003;27:1302-12.

2. Grossman K, Beasley MB, Braman SS. Hepatoid adenocarcinoma of the lung: Review of a rare form of lung cancer. Respir Med 2016;119:175-9.

3. Kelly PJ, Spence R, Dasari BV, et al. Primary hepatocellular carcinoma of the pancreas: a case report and review of the heterogeneous group of pancreatic hepatoid carcinomas. Histopathology 2012;60:1012-5.

4. Nakashima H, Nagafuchi K, Satoh H, et al. Hepatoid adenocarcinoma of the gallbladder. J Hepatobiliary Pancreat Surg 2000;7:226-30.

5. Sakamoto K, Monobe Y, Kouno M, et al. Hepatoid adenocarcinoma of the gallbladder: Case report and review of the literature. Pathol Int 2004;54:52-6.

6. Sakamoto K, Kimura N, Tokumura H, et al. Hepatoid adenocarcinoma of the gallbladder. Histopathology 2005;47:649-51.

7. Gakiopoulou H, Givalos N, Liapis G, et al. Hepatoid adenocarcinoma of the gallbladder. Dig Dis Sci 2007;52:3358-62.

8. Koswara T, Marwoto W, Siregar NC, et al. Hepatoid carcinoma of the gallbladder. Acta Med Indones 2007;39:179-82.

9. Wang Y, Sun L, Li Z, et al. Hepatoid adenocarcinoma of the stomach: a unique subgroup with distinct clinicopathological and molecular features. Gastric Cancer 2019;22:1183-92.

Cite this article as: Qian X, Zhou D, Gao B, Wang W. An alpha-fetoprotein-negative hepatoid adenocarcinoma of the gallbladder with squamous differentiation. Hepatobiliary Surg Nutr 2020;9(1):116-118. doi: 10.21037/hbsn.2019.12.03 